\title{
CREACIÓN DE LA CONSULTA DE ENFERMERIA PEDIÁTRICA EN LOS CENTROS INFANTILES DE LA ASOCIACION DE ROBLEALTO ${ }^{1}$
}

Institución: Centros Infantiles de la Asociación Roblealto

\author{
Viriam Leiva Díaz ${ }^{2}$ \\ Mónica González Quirós ${ }^{3}$
}

\section{COMO CITAR}

Leiva,V.; González, M. (2012).Creación de la consulta de Enfermería Pediátrica en los Centros Infantiles de la Asociación de Roblealto. [En línea].Rev. Enfermería Actual en Costa Rica, 22, 1-14 [citado (fecha)]. Disponible World Wide Web: <http://www.revenf.ucr.ac.cr/roble.pdf> ISSN $1409-4568$

\section{RESUMEN}

Esta investigación tuvo como objetivo analizar el aporte obtenido al crear la consulta de Enfermería Pediátrica que se ofrece a los niños, niñas y preadolescentes de alto riesgo de los centros infantiles de la Asociación Roblealto. Inicialmente, se diagnosticaron las necesidades de la población en estudios para, luego, poder implementar la consulta de Enfermería Pediátrica en tres centros infantiles con el fin de evaluar su respectivo funcionamiento. Este trabajo fue una investigación aplicada, con un enfoque mixto. Entre los principales hallazgos se mencionan las necesidades educativas que tienen las madres que reciben el servicio en los centros infantiles en relación con el cuidado de sus hijos e hijas, razón por la cual requerían de un profesional en el área de Enfermería Pediátrica para identificar e intervenir en las dificultades que puedan limitar el adecuado crecimiento y desarrollo de los infantes y preadolescentes. Al finalizar la consulta de Enfermería Pediátrica en los centros infantiles se evidencia la viabilidad de la intervención, ya que se observaron resultados positivos en los niños, niñas, preadolescentes y sus familias, por otra parte, la propuesta responde favorablemente a las políticas de salud en la atención del menor de edad en riesgo social. Conclusión: tras la consulta, se obtuvieron resultados positivos en los niños, niñas, preadolescentes y sus familias, ya que el recurso especializado representa un excelente medio para asesorar en cuanto a la educación en salud.

Palabras clave: Enfermería-pediátrica, niñez, riesgo-social

\footnotetext{
${ }^{1}$ Fecha de recepción: 10 de agosto del 2011

Fecha de aceptación: 15 de noviembre del 2011

${ }^{2}$ Viriam Leiva Díaz: Profesora catedrática de la Escuela de Enfermería de la Universidad de Costa Rica. Enfermera, psicóloga, Magister en Psicopedagogía investigadora del Programa de Investigación en enfermería y del Instituto de Investigaciones Filosóficas. Correo electrónico: viriaml@gmail.com / viriam.leiva@ucr.ac.cr

${ }^{3}$ Enfermera, Magister en Enfermería Pediátrica, Asociación Roblealto. Correo electrónico: mogonzalez78@hotmail.com
} 


\title{
CREATION OF PEDIATRIC NURSING CONSULTATION ON THE CHILDREN'S CENTER OF ROBLEALTO $^{1}$
}

\author{
Viriam Leiva Díaz ${ }^{2}$ \\ Mónica Gónzález Quirós ${ }^{3}$
}

\begin{abstract}
Institution: Children's Center of Roblealto CITED
\end{abstract} Actual en Costa Rica, 22, 1-14 [cited (date)]. Available World Wide Web: <http://www.revenf.ucr.ac.cr/roble.pdf> ISSN 1409-4568

The objective of this research was to analyze the contribution obtained by creating the Pediatric Nursing consultation offered to children and tweens at high risk of early childhood centers Roblealto Association. Initially, we diagnosed the needs of the population, then, to implement the Pediatric Nursing consultation in three children's centers to evaluate their respective performance. This work was applied research, with a mixed approach. Among the major findings are mentioned educational needs who have mothers who are served in childcare centers in relation to the care of their children, reason require a professional in the field of Pediatric Nursing to identify and intervene the difficulties that may limit the proper growth and development of infants and tweens. At the end of Pediatric Nursing consultation in child care centers is evidence of the viability of the intervention as positive results were observed in children, preteens and their families, on the other hand, the proposal responds favorably to health policy the attention of the child at risk. Conclusion: After the consultation is obtained positive results in children, preteens and their families, as the specialized resource is an excellent way to give advice about health education.

Keywords: childhood, Pediatric-nursing, social-risk.

\footnotetext{
${ }^{1}$ Date of reception: August 10, 2011

${ }^{2}$ Viriam Leiva Díaz: Profesora catedrática de la Escuela de Enfermería de la Universidad de Costa Rica. Enfermera, psicóloga, Magister en Psicopedagogía investigadora del Programa de Investigación en enfermería y del Instituto de Investigaciones Filosóficas. E-mail: viriaml@gmail.com / viriam.leiva@ucr.ac.cr

${ }^{3}$ Enfermera, Magister en Enfermería Pediátrica, Asociación Roblealto. E-mail: mogonzalez78@hotmail.com
}

Date of acceptance: November 15, 2011 


\section{INTRODUCCION}

El acceso a los servicios de salud es un derecho de todas las personas en general y, en especial, de la población infantil. En Costa Rica, este derecho no se refleja de forma equitativa, debido a que la población marginal obtiene poco o nada de dicho beneficio. Al respecto, el Fondo de las Naciones Unidas para la Infancia (UNICEF, 2008) en el VI estado de los Derechos de la niñez y la Adolescencia en Costa Rica menciona que "la protección integral está lejos de garantizar los derechos de las personas menores de edad únicamente por medio de una ley; se requiere de un sistema de protección social adecuado, que le dé integralidad y que permita la exigibilidad, vigilancia y cumplimiento de derechos" (p.8)

Es evidente que un aspecto fundamental para la persona menor es la salud, por ello la Organización Panamericana de la Salud (OPS) estableció los parámetros de las políticas y las directrices en esta materia para mantener un adecuado desarrollo humano creando servicios de salud, así como fortaleciendo la higiene en los diversos medios de vida, la prevención y lucha contra las enfermedades (Magán, I.; Vicente, A.; Berdullas, S. 2010)

En el 2009, el PANI y la UNICEF definieron como una política institucional prioritaria el cuidado de la niñez temprana, definiéndola como un período que requiere no solo de cuidados para asegurar su conservación, crecimiento físico y la protección contra las enfermedades, sino como uno que requiere de desarrollo cognitivo y psicosocial de toda la población infantil, sin que exista ningún tipo de discriminación, además de que debe garantizar la satisfacción de sus necesidades y otorgar prioridad a la adquisición de habilidades que han demostrado ser claves para la vida, dentro de las cuales están las habilidades lingüísticas, el desarrollo de la inteligencia y las capacidades socio-afectivas, las cuales potencian la creatividad, la actitud crítica y la construcción del conocimiento como parte del derecho que tiene cada niño y niña.

En los centros infantiles de la Asociación Roblealto, ingresan niños y niñas con diferentes factores de riesgo, sociales, psicológicos, ambientales y económicos; al respecto, en el 2007 se documenta que se atendieron 395 menores y 254 familias que presentan riesgo social, a quienes se atiende por distintas razones entre ellas: inestabilidad emocional, problemas de conducta, abandono y negligencia por parte de los progenitores, abuso sexual; agresión, discapacidad, antecedentes psiquiátricos, enfermedades crónicas y alcoholismo, entre otros. De igual forma, otro factor que afecta la calidad de vida del grupo familiar es el bajo ingreso económico, ya que el 95\% cuenta con un ingreso neto de 280.000 colones y en el 5\% de las familias es superior a 300.000 colones. (Perfil de las familias: Perfil de niños y niñas de la Asociación Roblealto, 2007)

La capacidad adquisitiva está relacionada con las ocupaciones que desempeñan los proveedores y las proveedoras, entre las que se destacan los servicios domésticos o trabajos como misceláneas, vendedoras ambulantes, operarias, ayudantes de cocina o dependientes, labores todas con una remuneración económica muy baja.

En relación con los barrios de los cuales proceden las familias que ingresan a los centros infantiles, estas son de los lugares aledaños a los centros, como San Francisco de Dos Ríos, Desamparados, Paso Ancho, Aserrí, Hatillo, San Sebastián, Alajuelita y 15 de Setiembre; algunas de ellas tienen de 2 a 4 hijos e hijas, por tanto, 
requieren los servicios diurnos brindados en Roblealto, pues solo así los encargados de los niños pueden ir a trabajar, máxime que en la mayoría de los casos predomina el tipo de familia uniparental, es decir, aquella conformada por una única figura de autoridad como padre, madre o encargado.

Respecto de la nacionalidad de la población atendida en los centros infantiles, un $47 \%$ es extranjera en de los casos, en su mayoría de nacionalidad nicaragüense, y un 53\% es costarricense. Aunado a la condición de inmigrante, se encuentra la condiciónde ilegalidad (57\%), condición que disminuye el poder optar por trabajos con una mayor remuneración y estabilidad económica.

Otro aspecto importante es el nivel de escolaridad de las familias, de las que un $2 \%$ no posee escolaridad, un $9 \%$ tiene primaria incompleta; un $29 \%$, primaria completa; un $36 \%$,secundaria incompleta, un $16 \%$, secundaria completa; un $6 \%$, universidad incompleta y un $2 \%$, universidad completa.

En cuanto al rubro salud, se determinó que entre un 2\% y 3\% de la población total atendida en los centros infantiles predomina la discapacidad física en los menores. Otros factores de riesgo evidenciados son la inestabilidad emocional, problemas de conducta, abandono o negligencia que requieren atención psicológica para atender sus dificultades. (Perfil de las familias: Perfil de niños y niñas de la Asociación Roblealto 2007)

Debido a lo anterior, fue fundamental que la Enfermera Pediátrica contextualice a las personas desde su entorno social y ambiental, pues ambos escenarios influyen en la salud y bienestar de la familia. La labor de la enfermera es primordial en la población infantil, dado que al identificar los problemas más frecuentes en la comunidad, facilitael apoyo en el área de salud pues integra al núcleo familiar en el proceso de Enfermería, sistema de apoyo y responsable de transmitir hábitos, creencias y valores a cada uno de los miembros.

El objetivo de este estudio fue analizar el aporte que brinda la creación de una consulta de Enfermería Pediátrica para los niños, niñas y preadolescentes de alto riesgo que se atienden en los centros infantiles de la Asociación Roblealto.

\section{MATERIALES Y METODO}

Como población meta del estudio se incluyó a los niños, niñas y preadolescentes de alto riesgo y a sus familias, atendidos en los tres centros infantiles. En total fueron atendidos doce niños, niñas y preadolescentes a quienes refirieron los referidosenfermeros y enfermeras de los centros respectivos.

De acuerdo con los propósitos del estudio, la investigación es aplicada, la cual según Vargas (2009) "implica un procedimiento llevado a cabo mediante encuestas, entrevistas o cuestionarios, para establecer las necesidades o problemas que afectan un sector o una situación de la realidad social y que es motivo de estudio o investigación.” (p.161). De esta manera la indagación responde a las necesidades e intereses del grupo, el cual se vería beneficiado si se implementara la consulta de Enfermería Pediátrica en los centros infantiles de la Asociación Roblealto.

Respecto del lugar en donde se llevó a cabo este trabajo, cabe decir que la asociación fue fundada en 1932; es una ONG de bienestar social, de carácter cristiano y sin fines de lucro, que brinda sus servicios a la infancia y a 
las familias con diversos factores de vulnerabilidad social como problemas económicos, de salud física y mental, maltrato infantil de algún tipo, problemas de conducta y académicos y viven violencia intrafamiliar sufrida por madres jefas de hogar que que además cuentan con pocos recursos económicos para superar su situación familiar.

El trabajo de la Asociación Roblealto se desarrolla a través de tres centros infantiles diurnos que atienden niños y niñas desde los 9 meses hasta los 12 años de edad. El primero es el Centro Infantil el Hogar del Niño Feliz ubicado en Barrio Naciones Unidas en San José.El segundo es el Centro Infantil 15 de Setiembre, ubicado en la localidad con el mismo nombre y, finalmente, el Centro Infantil El Manantial, localizado en la carretera a Desamparados.

Cada institución cuenta con los servicios profesionales de enfermería, medicina, psicología, trabajo social, asistencia educativa, asistencia de educación cristiana, así como de comedor, en el cual se brinda una alimentación realizada y supervisada por una nutricionista; además, hay actividades recreativas y educativas, traslado a la escuela, entre otros.

Para la implementación de la consulta, se solicitó el permiso respectivo a la Asociación Roblealto,. Luego, para seleccionar los criterios de inclusión o referencia y la documentación elaborada para tal fin, se realizó una reunión con los enfermeros y enfermeras de cada centro infantil.

Para realizar el diagnóstico de las necesidades, se aplica un cuestionario a los profesionales de enfermería y a la supervisión de enfermería para valorar la importancia del desarrollo de la consulta de la Enfermería Pediátrica y determinar qué casos necesitan ser atendidos y con qué prioridad.

En la consulta se realiza una entrevista inicial con las madres y padres de los niños, niñas y adolescentes, con el fin de explicarles el propósito de la consulta de enfermería pediátrica.

Después, se procedió a aplicar los instrumentos evaluativos tales como historial de enfermería, Test de Tanner, Test de Denver II, APGAR familiar I y II, examen físico, valoración de peso y talla. Lo anterior permitió identificar los factores de riesgo en los niños, niñas y preadolescentesa partir de que se elabora el plan de intervención según las necesidades de cada caso, incluyendo a los padres, madres y encargados en el proceso.

Finalmente, se lleva a cabo la etapa de evaluación, la cual se realiza al culminar la intervención de la consulta; para realizarla se empleó un cuestionario con preguntas abiertas y cerradas aplicado a las familias, niños, niñas y preadolescentes atendidos y a los profesionales de Enfermería.

\section{CONSIDERACIONES ETICAS}

En la investigación se consideraron principios bioéticos tomados de Blázquez (2004), entre los que se pueden mencionar la autonomía, pues se respetó a las personas como individuos libres y se tuvo en cuenta en todo momento sus decisiones; la veracidad, esto por cuanto, tanto a los infantes participantes como a sus padres o encargados se les informó verazmente sobre todo lo referente a su participación en la consulta. Se mantuvo la privacidad mediante el anonimato de las personas participantes. Finalmente, se aseguró el principio de Beneficencia y no maleficencia, mediante la aplicación de actividades que no fueran en contra de los principios, 


\section{Revista Electrónica Enfermería Actual en costa Rica}

sentimientos, creencias o integridad de cada participante; además se buscó su beneficio, por medio de la construcción de conocimientos respecto del tema de interés de las consultantes.

\section{RESULTADOS}

Para enmarcar los resultados, se consideró importante indagar la opinión de los profesionales de Enfermería que laboran en los centros respecto decuán importante es la apertura de una consulta de Enfermería Pediátrica, a lo la totalidad afirmó que es muy importante.Por otro lado, se exploró el perfil laboral que, a criterio de los encuestados, debe poseer el o la enfermera pediátrica dentro de la organización. En relación con lo anterior se destacan los siguientes puntos:

- En el primer nivel de atención se debe brindar seguimiento a niños, niñas y adolescentes de los centros.

- Brindar educación para la salud y cuidados preventivos a los niños, niñas, adolescentes y sus familias.

- Brindar tamizaje de crecimiento y desarrollo de los menores.

- Supervisar la seguridad e higiene de las aulas y cocinas, alimentación y cuidados básicos para que los menores de edad se desenvuelvan en un ambiente saludable.

- Coordinar la Brigada de Primeros Auxilios y el Plan de Emergencia de cada centro infantil.

Cabe destacar que, tomando en cuenta los aspectos socioeconómicos de las familias, así como el principio de oportunidades, se decidió realizar la consulta de Enfermería Pediátrica itinerante, es decir, se atendía a los menores en un centro de la Asociación Roblealto que le correspondía.

Entre los aspectos que se tomaron en cuenta para fijar los horarios se encontraban: la posibilidad de los padres y madres de que pudieran asistir a la consulta, sin que esto significara sacrificar tiempo laboral y que coincidiera con el horario de salida de los centros, lo cual también permitió hacer uso de las instalaciones físicas para el desarrollo de la consulta.

Seguidamente, se procedió a identificar los casos referidos. En el Centro Infantil Hogar del Niño Feliz se identificaron tres niños y dos niñas menores de 9 años en quienes se detectó cuadros asociados a retraso en el desarrollo psicomotor, riesgo nutricional y familiar. En el Centro Infantil 15 de Setiembre se refirieron tres niñas, dos niños y un preadolescente, cuyas edades oscilan entre los 3 años y 10 años de edad y presentan diagnósticos similares a los mencionados; por último, el Centro Infantil el Manantial fue el sitio que realizó mayor cantidad de referencias, pues ahí porvienen 11 niñas y niños con edades desde los 2 hasta los 11 años de edad con diagnósticos asociados a trastornos psicomotores severos, riesgos nutricionales, enfermedades crónicas y discapacidad.

Con los casos referidos, se procedió a implementar la consulta de Enfermería Pediátrica que se diseñó de acuerdo con las necesidades de la población, la disponibilidad de esta y del espacio físico.

Para las sesiones se utilizaron los espacios de la consulta de los enfermeros y las enfermeras de los centros, los cuales cuentan con equipo y mobiliario que facilitó el espacio idóneo para la consulta de Enfermería Pediátrica. 
La población atendida reside fundamentalmente en los alrededores de los centro infantiles a los que asisten. Las cuidadoras (11) son en su mayoría madres de los niños, niñas y preadolescentes atendidos; sus edades oscilaban entre los 20 y 35 años.

Las consultas se realizaron en una sesión por semana, con una duración entre 35 a 60 minutos aproximadamente, las cuales culminaron en agosto de ese mismo año.

Se realizaron una totalidad de 11 consultas por menor de edad y familia, las cuales se detallan de la siguiente manera:

- La primera consulta tuvo como finalidad conocer a las familias y establecer un vínculo con ellas. Además se inicia explicando la metodología, tiempo, y horario por seguir durante la consulta. Se les expresó detalladamente lo que se esperaba de ellos y ellas; una vez satisfechas sus dudas, se procedió a la firma del consentimiento informado.Durante esta consulta expresaron las siguientes expectativas:

-"Necesito ayudar a mi hija a bajar de peso" (p2)-

-“Quiero saber si mi hijo tendrá esta enfermedad siempre y por qué pasó" (p11)-

-"No sé cómo ayudar a mi hija. Yo trato de hacerle todo, pero no sé si está bien.”(p9)-

-“Quiero que mi hijo se pueda comunicar mejor” (p3)-

A partir de esta consulta se obtuvo información muy valiosa para la investigación, entre la que se encuentralas expectativas de cada cuidadora y datos personales. Sin embargo, el principal provecho fue para la cuidadora, porque a partir de este espacio puede construir una mejora en el cuidado que brinda.

- En la segunda consulta se procede a diagnosticar los casos referidos, iniciando con la aplicación del Historial de Enfermería para evaluar la condición de vida de los beneficiarios, así como los factores de riesgo que afectan la salud integral de los menores de edad y su familia.

En cuanto a antecedentes patológicos personales y familiares se destacaron: asma en dos menores, diabetes mellitus en una de las cuidadoras, dos madres con sobrepeso y tres con obesidad de grado II. Además, las progenitoras mencionaron su falta de conocimiento en hábitos alimenticios y de recreación.

Respecto de las condiciones de vida, se encontró que las familias atendidas se caracterizaron como de bajo nivel económico y baja escolaridad, ya que dos de las madres atendidas no saben leer ni escribir, una tiene secundaria completa y las ocho restantes tan sólo tienen primaria completa.

Asimismo, en cuanto a la tenencia de vivienda, nueve de las familias alquilan casa y dos de ellas viven con la familia materna. Al mismo tiempo, se identificaron tres familias de tipo nuclear tradicional (papá o mamá), cuatro de la categoría extensa (abuelos maternos, tíos maternos) y cuatro de un solo progenitor. De las anteriores, ocho de ellas son madres jefas de hogar.

Del mismo modo, se observó que en la totalidad de las familias evaluadas existe comprensión de los problemas presentes en sus hijos e hijas y que requieren un cambio de actitud y de modificaciones en sus hábitos. 
- En la tercera consulta, se realizó examen físico, medición de peso/talla y aplicación del test de Tanner a los y las preadolescentes; además, se aplicó el test de desarrollo Denver a los niños y niñas menores de 6 años.

En la valoración de peso y talla realizada, se encontró que tres infantes tienen sobrepeso y dos, obesidad, mientras que un niño y un preadolescente estaban en desnutrición leve y cinco se encontraban con peso y talla normal para sus edades.

Por otro lado, en la valoración de desarrollo a los niños y niñas menores de 6 años, se evidencia que dos de ellos y ellas presentaban retraso en su desarrollo, mientras que ocho presentaban adecuado progreso psicomotor para su edad cronológica. Sin embargo, se refleja una carencia en estimulación del desarrollo para la vida, especialmente en motora gruesa y lenguaje, debido a que las cuidadoras expresaban su falta de conocimiento para realizarlo y el tiempo disponible para ello. Igualmente, se requirió aplicar el test de Tanner a dos preadolescentes, quienes se muestran en bajo riesgo y con adecuada madurez sexual.

Por otro lado, en la cuarta consulta se aplicó el test de APGAR familiar y se evidencia que nueve familias se evaluaron como altamente funcionales y en bajo riesgo y dos familias como medianamente funcionales y en bajo riesgo.

Entre los factores de riesgo encontrados en la fase diagnóstica de la consulta de Enfermería Pediátrica se encontraron los siguientes: Sobrepeso, desnutrición, retraso en el desarrollo psicomotor, problemas emocionales, alteraciones psiquiátricas, enuresis nocturna, falta de conocimiento en manejo de disciplina, hábitos alimenticios, necesidades recreativas, desconocimiento de los problemas presentes de sus hijos e hijas y de los recursos que brinda la instancias de la C.C.S.S., asma, dermatitis atópica, Diabetes Mellitus e inexistencia de medidas para la resolución de los problemas.

- A partir de los resultados mencionados hasta aquí, desde la quinta consulta, hasta la décima consulta, el fin fue implementar un plan de atención basado en las necesidades de cada uno de los menores y sus familias, cuyo abordaje se daría de manera paulatina.

El plan de intervención se apoyó en el sistema sustentador educativo (Orem,1983) en temas como alimentación y ejercicio, técnicas para mejorar la enuresis nocturna, cuidados en enfermedad crónicas, hábitos de higiene personal y ambiental, estimulación temprana, técnicas para el manejo de límites, técnicas de estudio, información en las leyes y derechos de los menores de edad, orientación en los recursos que ofrece la C.C.S.S., además se realizó un taller llamado "Conozco los grupos de alimentos y sus beneficios" para niños y niñas con problemas nutricionales.

Entre los resultados de la evaluación, última fase del estudio, se obtuvieron los siguientes:

En cuanto a los materiales utilizados, las familias manifestaron que les gustó mucho, porque fue hecho acorde con las necesidades e intereses de sus hijos e hijas. También consideraron que el tiempo empleado en la intervención fue adecuado, dado que se atendieron las dificultades presentadas durante el proceso de la consulta. 


\section{Revista Electrónica Enfermería Actual en costa Rica}

El espacio utilizado para la consulta fue adecuado, tanto para los profesionales como las cuidadoras, puesto que no tenían que trasladarse a ningún otro lugar.

En cuanto a la relación de la enfermera con la cuidadora, niños, niñas y preadolescentes, los participantes mencionaron que estaban satisfechos, ya que la totalidad de las familias intervenidas aludieron que la consulta de Enfermería Pediátrica les ha servido para ayudar a sus hijos e hijas. Por otro lado, mencionaron que les aportó beneficios para mejorar la situación de sus hijos e hijas tales como promover hábitos alimenticios tanto en los menores como en el resto de la familia, cuidados especiales en los problemas de asma y dermatitis, conocimientos de los padecimientos de los niños y niñas, mayor comprensión de los problemas de salud de los menores de edad, así como estimular a los infantes según su etapa de desarrollo.

De este modo, los progenitores opinaron que sería excelente que la consulta de Enfermería Pediátrica permaneciera abierta, ya que otros niños y niñas mejorarían su estado de salud. En el caso de este estudio, tanto las familias, como los infantes y preadolescentes de la Asociación Roblealto se vieron beneficiados, sin necesidad de pagar o invertir tiempo para trasladarse a otros lugares que no correspondan a los centros educativos a los que asisten.

Asimismo, la totalidad de los niños, niñas y preadolescentes atendidos opinaron que se sentían muy bien con el trato que les brindó la Enfermera durante la consulta y la totalidad de ellos y ellas manifestaron haberse sentido muy bien en la intervención de la consulta de Enfermería Pediátrica.

\section{DISCUSION}

Las poblaciones que se atienden en los centros de Roblealto, en su mayoría, son de alto riesgo socioeconómico, por tanto, requieren intervenciones especializadas que den seguimiento al desarrollo de la población infantil para de esta forma servir de guía para las familias, para que ellos mismos puedan contribuir con dicho desarrollo evolutivo (Orem, 1983)

Por lo anterior, en esta investigación se tomó en cuenta las necesidades de menores de edad y sus familias, ya que como lo establece el Manual de Enfermería (Océano, 2007):

La Enfermera o el enfermero: toma en consideración todas las dimensiones del individuo y su entorno; se debe tener en cuenta, pues, las necesidades fisiológicas, psicológicas, sociales, culturales y espirituales del ser humano. Cualquier factor que impida o dificulte la satisfacción de tales necesidades, ya sea interno (individual) o bien externo (ambiental), priva al individuo de su total autonomía y puede requerir una actuación de Enfermería destinada al restablecimiento de la salud en su sentido más amplio. (p.1)

De ahí, la importancia de la coordinación con estos profesionales en enfermería que laboran en los centros, ya que ellos y ellas conocen muy bien las limitaciones y necesidades que enfrentan los menores y las familias que atienden. 
En este sentido, cabe destacar la preocupación expresada por los profesionales respecto de situaciones que requieren de un seguimiento continuo y especializado y que están fuera de su ámbito de acción profesional, en los cuales un profesional en el área de Enfermería Pediátrica podría intervenir oportunamente proporcionando seguimiento individualizado según las necesidades de esta población. Lo anterior coincide con lo que apuntan Chávez, Gutiérrez y Solano (2006): "Los especialistas en Enfermería Pediátrica son los indicados para asumir las consultas de educación y seguimiento a los niños, niñas y población adolescente con carencias especiales”(p.70)

En la implementación de la consulta se mantuvo la horizontalidad entre los y las personas responsables y la enfermera, lo cual propicióun entorno favorable para la expresión de experiencias de vida, situaciones cotidianas, preocupaciones, dudas, y el abordaje de los aspectos de mayor interés para los y las cuidadoras(Instituto Nacional para la Educación de Adultos de México, 2007)

Cabe señalar que al inicio de la consulta de Enfermería Pediátrica las cuidadoras se encontraban en la fase de la orientación; según lo planteado por Peplau (1990), esta fase "inicia cuando las personas consideran que necesitan ayuda para enfrentar un problema y recurren en busca de orientación profesional” (p.17). Por consiguiente, en esta etapa se intenta averiguar, tanto como fue posible, las limitaciones de cada situación específica de cada menor, su familia y su entorno con el fin de identificar sus necesidades, problemas e inquietudes. De ahí que en esta primera intervención, las cuidadoras manifestaran la necesidad de herramientas y conocimientos para apoyar a sus hijos e hijas; asimismo, se evidencia que algunas madres expresaron sentimientos de culpabilidad en relación con los problemas presentes en los menores (Sánchez, Valencia, 2007)

Comprendiendo que la comunicación tiene un carácter fundamentalmente indicativo de mensajes implícitos referidos a datos personales, al contexto, a deseos y expectativas individuales, se implementaron actividades que favorecieron la interacción entre los cuidadores y las cuidadoras y la enfermera pediátrica, rescatándose el papel que deben desempeñar la enfermera pediátrica para clasificar y propiciar la comunicación entre las personas participantes (Leirman, col., 1991)

En cuanto al aspecto económico, cabe destacar lo encontrado por Flores, Puntel y Castillo (2006) en su investigación, en la cual al no darse lo anterior, la gente no pudo asistir a la consulta, de allí la novedad de el tipo de consulta implementada en este trabajo (consulta itinerante), en la cual es el profesional quien se desplaza y no los consultantes. La participación del grupo conincide con lo expresado por Rojas y Leiva (2009) en cuanto a que "el concepto de autocuidado refuerza la participación activa de las personas en el cuidado de su salud como responsable de decisiones que condicionan su situación”. (p. 57), pero para llevar a cabo tales acciones se debe tener presente las necesidades socioeconómicos que pueden intervenir en el cuidado del niño, niña y preadolescente.

Es importante señalar que ocho de las madres son jefas de hogar, lo cual coincide con el Decimosexto Informe de la Nación en el Desarrollo Humano Sostenible (Programa Estado de la Nación, 2010) que menciona que la estructura de la familia en Costa Rica ha cambiado del esquema tradicional de hogares biparentales a los monoparentales con jefatura femenina, transformación que a su vez se asocia a mayor pobreza.

Además, en la presente investigación se encontró que en la totalidad de las familias existe comprensión de los problemas presentes en sus hijos e hijas y que requieren de un cambio de actitud y de modificaciones en sus 
hábitos. Esto concuerda con lo manifestado por Morales (2008) y Mondol (2006), quienes notaron en sus investigaciones que las madres requieren orientación, apoyo y seguridad. De ahí se puede constatar la necesidad de actualizar y potenciar las habilidades de las personas en cuanto a hábitos alimenticios, técnicas de estimulación, disciplina, autocuidado y recursos existentes en el área infantil.

Un factor protector que se une al éxito de las necesidades detectadas es que se evidenció que nueve las familias son altamente funcionales y dos medianamente funcionales, según APAGAR familiar (Forero y cols, 2006), lo que fue de importancia para la investigación, ya que la situación familiar y la relación entre cada uno de los miembros de familia puede ayudar a los profesionales en Enfermería a evidenciar su relación con el ámbito de la salud y lograr mayor posibilidad de éxito (Aguilar, 2007)

La valoración de crecimiento y desarrollo de los niños, niñas y preadolescentes participantes fue fundamental, pues como lo mencionan Hoekelman (2000) y Salas (2008), esta valoración es crucial, ya que cualquier desviación de la norma constituye una advertencia precoz de la existencia de un proceso patológico; al respecto, en la presente investigación se encontró retraso motor en algunos de los infantes, producto de la falta de conocimiento para realizarlo y el tiempo disponible para ello, según las cuidadoras.

Al finalizar la fase diagnostica se evidenciaron otras necesidades que requerían intervención, lo que permitió implementar el plan de Enfermería de forma individualizada, lo cual corresponde a lo señalado por Aguilar (2007): "La ejecución de proceso de Enfermería consiste en ejecutar las actividades de Enfermería, registrar los cuidados administrados y mantener un plan individualizado (p19). Por tanto, estas actividades estuvieron dirigidas a prevenir, promover y mantener o restablecer la salud de los menores y sus familias.

En este proceso investigativo se tomaron en cuenta los derechos de los niños, niñas y adolescentes, lo cual concuerda con lo propuesto por Quirós (2006), cuando afirma que

Los derechos humanos buscan garantizar el bienestar, una vida digna para todas las personas, en su dimensión individual, social, material y espiritual sin discriminación por razón de etnia, religión, nacionalidad, condición económica, edad, sexo, escolaridad, preferencia sexual y condición de salud. (p.1)

Lo anterior se realizó a través de la educación brindada a las cuidadoras y la participación de los menores en diferentes aspectos de su autocuidado (López y Sarrate, 2002)

Asimismo, este proyecto valoró las diferentes necesidades de los menores de edad de manera individual, coincidiendo con la OMS (Unicef, 2001):

La vulnerabilidad de los niños y niñas varía según la edad y los dinámicos procesos de crecimiento y desarrollo. Los efectos de la exposición pueden ser, por ejemplo: durante el desarrollo prenatal o al nacer, aborto espontáneo, muerte prenatal, insuficiencia ponderal al nacer o malformaciones congénitas; en niños pequeños, mortalidad infantil, asma, deficiencias neurocomportamentales o inmunitarias; y en los adolescentes pubertad precoz o retrasada. (p.1) 
Por consiguiente, en este estudio fue vital tomar en cuenta alternativas de protección del menor que permitan su adecuado desarrollo y que procuren cuidados en un ambiente saludable tanto para los niños, niñas y preadolescentes como para sus familias.

\section{CONCLUSIONES}

- Se visualiza la viabilidad del proyecto en las tres instituciones, ya que existe la necesidad de crear espacios para los niños, niñas y adolescentes de alto riesgo y se se puede dar seguimiento no solo al menor de edad, sino también al núcleo familiar.

- Los padres y madres integrados en la consulta de Enfermería Pediátrica lograron conocer la problemática de sus hijos e hijas, a partir de lo que crearon estrategias para lograr cambios oportunos y mejorar la situación de salud.

- El acercamiento con los padres, madres de los niños, niñas y preadolescentes, favoreció la intervención de la Enfermera Especialista, ya que lograron externalizar sus preocupaciones y sentimientos respecto de la situación de sus hijos e hijas, colaboración que logró mejorar las debilidades en su cuidado.

- Tras la consulta se evidenció resultados positivos en los niños, niñas, preadolescentes y sus familias, ya que el recurso especializado representa un excelente medio para la prevención de la educación en salud.

- La creación de una consulta de Enfermería Pediátrica fue de gran beneficio, principalmente para los casos de mayor necesidad de atención, además de que representó un gran apoyo para el equipo de salud de la Asociación Roblealto. Con esta intervención se logró incluir al grupo familiar para darle seguimiento al Proceso de Enfermería y, así, brindar mayores beneficios.

\section{REFERENCIAS}

Aguilar, C. (2007). Tratado de Enfermería Infantil, Cuidados Pediátricos. Madrid, España: Editorial Océano.

Asociación Roblealto, Pro Bienestar del Niño (2007). Perfil de niños (as) y familias de los Centros Infantiles, Hogar biblico y programas de adolescentes. San José, Costa Rica: Roble Alto.

Blázquez, N. (2004). La Bioética y los Hijos del Futuro. Madrid, España: Visión NET.

Chávez, D., Gutiérrez, I., Solano, F. (2006). Implementación de Consultas de Enfermería Pediátrica dirigida a niños, niñas y población adolescente asmática en clínicas del área metropolitana y Esparza: Caja Costarricense de Seguro Social.Trabajo Final de Graduación no publicada, Universidad de Costa Rica. San José, Costa Rica.

Flores, Y., Puntel, M., Castillo, R. (2006). El proceso del trabajo de la enfermera en el cuidado del niño sano en una institución de la seguridad social de México. Recuperado de http://www.scielo.br/pdf/rlae/v14n5/es v14n5a03.pdf

Forero, L., Avendaño, M., Duarte, Z., Campo, A. (2006). Consistencia interna y análisis de factores de la escala APGAR para evaluar el funcionamiento familiar en estudiantes de básica secundaria. Revista Colombiana de Psiquiatría, 35(1):2329. Recuperado de http://redalyc.uaemex.mx/src/inicio/ArtPdfRed.jsp?iCve $=80635103$ 


\section{Revista Electrónica Enfermería Actual en costa Rica}

Hoekelman, A. (2000). Atención Primaria en Pediatría. Barcelona, España: Editorial Océano.

Instituto de las mujeres de la Ciudad de México (2007). Andragogía. Recuperado de http://tecnoeduka.110mb.com/documentos/teoría\%20aprendizaje/Andragogia\%202.pdf

Leirman, W., Vndemeulebroecke, L., Baert, H., Callens, H., Wildemmeersch, D. (1991). La educación de adultos como proceso. Madrid, España: Editorial Popular, S.A.

López, E. y Sarrate, M. (2002) La Educación de las Personas Adultas: Reto de Nuestro Tiempo. Madrid, España: Dykinson.

Magán, I.; Vicente, A.; Berdullas, S. (2010). Salud Mental en Niños y Adolescentes. Traducción de The mental health of children and adolescents. Conclusiones de la conferencia de la OMS realizada en septiembre de 2004 en Luxemburgo.Organización Mundial de la Salud (WHO) (2004). Recuperado de http://www.cop.es/infocop/pdf/1861.pdf

Mondol, J. Consulta de Enfermería pediátrica para la niñez y adolescencia con asma de Esparza. Rev. Enfermería Actual en Costa Rica [en línea]. Abril-Setiembre No.12 [citado (2007)]. Disponible World Wide Web: < http://www.revenf.ucr.ac.cr/asmainfantil.pdf> ISSN 1409-4568.

Morales, H. (2008) Conocimiento de madres adolescentes sobre el cuidado del niño y el rol materno. Recuperado de http://www.portalesmedicos.com/publicaciones/articles/1126/1/Conocimiento-que-tienen-las-madres-adolescentes-sobre-elcuidado-del-nino-y-su-relacion-con-el-desempeno-del-rol-materno.html.

Oceáno (2007) Manual de Enfermería. Barcelona, España: Editorial Océano.

PANI, Unicef (2009) Política Nacional para la niñez y la Adolescencia Costa Rica: 2009- 2012. Recuperado de http://www.unicef.org/costarica/docs/cr_pub_Politica_NNA_CR.pdf

Orem, D. (1983) Normas prácticas en enfermería. Traducido por Susan Allen. Madrid, España: Ediciones Pirámide, S.A.

Peplau, H. (1990) Relaciones interpersonales en enfermería: un marco de referencia conceptual para la enfermería psicodinámica. Barcelona, España: Salvat.

Programa Estado de la Nación. (2010) Décimo Sexto Informe Estado de la Nación en Desarrollo Humano Sostenible. San José, Costa Rica. Recuperado de http://www.estadonacion.or.cr/index.php/biblioteca-virtual/costa-rica/estado-de-lanacion/sinopsis/informe-xvi

Quirós E. (2006) Algunas definiciones con los Derechos Humanos en Salud. San José, Costa Rica: Ministerio de Salud.

Rojas, L., Leiva, V. (2009) Modelo Conceptual de Imogene King y Dorothea Orem. San José, Costa Rica: Editorial Universidad de Costa Rica.

Salas, R. (2008) Estilos de Aprendizaje a la Luz de la Neurociencia. Bogotá, Colombia: Editorial Magisterio.

Sánchez, M, Valencia, S. (2007) Lectura Sistémica Sobre Familia y el Patrón de la Violencia. Bogotá, Colombia: Universidad de Caldas.

UNICEF (2001) Experiencias significativas del Desarrollo Infantil Temprano en América Latina y el Caribe. Ciudad Panamá, Panamá: Editorial Unicef. 


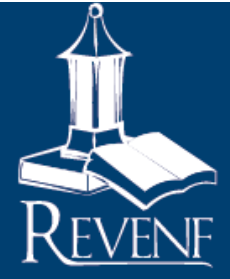

UNICEF (2008). VI informe del Estado de los derechos de la niñez y la Adolescencia en Costa Rica: a diez años del Código de la Niñez y la Adolescencia. San José, Costa Rica: Universidad de Costa Rica.

Vargas, Z. (2009). La investigación Aplicada: una forma de conocer las realidades con evidencia científica. Revista Educación de la Universidad de Costa Rica, 33(1), 155-165. 